\title{
Symmetric Division Deg Energy of a Graph
}

\author{
K. N. Prakasha ${ }^{1}$, P. Siva Kota Reddy ${ }^{2}$, Ismail Naci Cangul ${ }^{3,}$ \\ ${ }^{1}$ Mathematics, Vidyavardhaka College of Engineering, Mysuru, India \\ ${ }^{2}$ Mathematics, Siddaganga Institute of Technology, Tumkur, India \\ ${ }^{3}$ Mathematics, Uludag University, Bursa, Turkey \\ *Corresponding author: ncangul@gmail.com
}

Received July 28, 2017; Revised August 29, 2017; Accepted September 22, 2017

\begin{abstract}
The purpose of this paper is to introduce and investigate the symmetric division deg energy $\operatorname{SDDE}(G)$ of a graph. We establish upper and lower bounds for $\operatorname{SDDE}(G)$. Also the symmetric division deg energy for certain graphs with one edge deleted are calculated.
\end{abstract}

Keywords: symmetric division deg index, symmetric division deg eigenvalues, symmetric division deg energy, $k$-complement, $k(i)$-complement, edge deletion

Cite This Article: K. N. Prakasha, P. Siva Kota Reddy, and Ismail Naci Cangul, "Symmetric Division Deg Energy of a Graph." Turkish Journal of Analysis and Number Theory, vol. 5, no. 6 (2017): 202-209. doi: 10.12691/tjant-5-6-2.

\section{Introduction}

Let $G$ be a simple graph and let $\left\{v_{1}, v_{2}, \ldots, v_{n}\right\}$ be the set of its vertices. Let $i, j \in\{1,2, \ldots, n\}$. If two vertices $v_{i}$ and $v_{j}$ of $G$ are adjacent, then we use the notation $v_{i} \sim v_{j}$. For a vertex $v_{i} \in V(G)$, the degree of $v_{i}$ will be denoted by $d\left(v_{i}\right)$ or briefly by $d_{i}$.

In mathematical chemistry, topological indices play an important role due to their countless applications. There are many topological indices such as Randić index, sum-connectivity index, atom bond connectivity index, Zagreb indices, etc. One of those numerical descriptors, the symmetric division deg index, is included in the list of 148 discrete Adriatic indices and is a very good predictor of total surface area of polychlorobiphenyls (PCB).

The symmetric division deg index of a graph $G$ is defined by

$$
R=R(G)=\sum_{i \sim j}\left(\frac{\min \left\{d_{i}, d_{j}\right\}}{\max \left\{d_{i}, d_{j}\right\}}+\frac{\max \left\{d_{i}, d_{j}\right\}}{\min \left\{d_{i}, d_{j}\right\}}\right) .
$$

The concept of the symmetric division deg index motivates one to associate a symmetric square matrix $S D D(G)$ to a graph $G$. The symmetric division deg matrix $S D D(G)=\left(S_{i j}\right)_{n \times n}$ is, by this reason, defined as

$$
S_{i j}=\left\{\begin{array}{l}
\frac{\min \left\{d_{i}, d_{j}\right\}}{\max \left\{d_{i}, d_{j}\right\}}+\frac{\max \left\{d_{i}, d_{j}\right\}}{\min \left\{d_{i}, d_{j}\right\}} \text { if } v_{i} \sim v_{j}, \\
0 \quad \text { otherwise. }
\end{array}\right.
$$

\section{The Symmetric Division Deg Energy of a Graph}

Let $G$ be a simple, finite, undirected graph. The classical energy $E(\mathrm{G})$ is defined as the sum of the absolute values of the eigenvalues of its adjacency matrix. For more details on energy of a graph, see [2,3].

Let $S D D(G)$ be the symmetric division deg matrix. The characteristic polynomial of $\operatorname{SDD}(G)$ will be denoted by $\phi_{S D D}(G, \lambda)$ and defined as

$$
\phi_{S D D}(G, \lambda)=\operatorname{det}(\lambda I-S D D(G)) .
$$

Since the symmetric division deg matrix is real and symmetric, its eigenvalues are real numbers and we label them in non-increasing order $\lambda_{1} \geq \lambda_{2} \geq \ldots \geq \lambda_{n}$. The symmetric division deg energy of $G$ is similarly defined by

$$
\operatorname{SDDE}(G)=\sum_{i=1}^{n}\left|\lambda_{i}\right| .
$$

This paper is organized as follows. In Section 3, we give some basic properties of symmetric division deg energy of a graph. In Section 4, symmetric division deg energy of some specific graphs are obtained. In Section 5, we find symmetric division deg energy of some complements of some specific graphs. In Section 6, the symmetric division deg energy for certain graphs with one edge deleted are calculated and finally in Section 7, some open problems are given.

\section{Some Basic Properties of Symmetric Division Deg Energy of a Graph}

Let us define the number $P$ as 


$$
P=\sum_{i<j}\left(\frac{d_{i}^{2}+d_{j}^{2}}{d_{i} d_{j}}\right)^{2} .
$$

Then we have

Proposition 3.1. The first three coefficients of the polynomial $\phi_{S D D}(G, \lambda)$ are as follows:

(i) $a_{0}=1$,

(ii) $a_{1}=0$,

(iii) $a_{2}=-P$.

Proof. (i) By the definition of the polynomial

$$
\phi_{S D D}(G, \lambda)=\operatorname{det}[\lambda I-S D D(G)],
$$

we get $\mathrm{a} 0=1$.

(ii) The sum of determinants of all $1 \times 1$ principal submatrices of $\operatorname{SDD}(G)$ is equal to the trace of $\operatorname{SDD}(G)$ implying that

$$
a_{1}=(-1)^{1} \times \operatorname{tr}(\operatorname{SDD}(G))=0 .
$$

(iii) By the definition, we have

$$
\begin{aligned}
(-1)^{2} a_{2} & =\sum_{1 \leq i<j \leq n}\left|\begin{array}{ll}
a_{i i} & a_{i j} \\
a_{j i} & a_{j j}
\end{array}\right| \\
& =\sum_{1 \leq i<j \leq n} a_{i i} a_{j j}-a_{j i} a_{i j} \\
& =-P .
\end{aligned}
$$

Proposition 3.2. If $\lambda_{1}, \lambda_{2}, \ldots, \lambda_{n}$ are the symmetric division deg eigenvalues of $\operatorname{SDD}(G)$, then

$$
\sum_{i=1}^{n} \lambda_{i}^{2}=2 P
$$

Proof. It follows as

$$
\begin{aligned}
\sum_{i=1}^{n} \lambda_{i}^{2} & =\sum_{i=1}^{n} \sum_{j=1}^{n} a_{i j} a_{j i} \\
& =2 \sum_{i<j}\left(a_{i j}\right)^{2}+\sum_{i=1}^{n}\left(a_{i i}\right)^{2} \\
& =2 \sum_{i<j}\left(a_{i j}\right)^{2} \\
& =2 P .
\end{aligned}
$$

Using this result, we now obtain lower and upper bounds for the symmetric division deg energy of a graph: Theorem 3.3. Let $G$ be a graph with $n$ vertices. Then

$$
\operatorname{SDDE}(G) \leq \sqrt{2 n P} .
$$

Proof. Let $\lambda_{1}, \lambda_{2}, \ldots, \lambda_{n}$ be the eigenvalues of $S D D(G)$. By the Cauchy-Schwartz inequality we have

$$
\left(\sum_{i=1}^{n} a_{i} b_{i}\right)^{2} \leq\left(\sum_{i=1}^{n} a_{i}^{2}\right)\left(\sum_{i=1}^{n} b_{i}^{2}\right) .
$$

Let $a_{i}=1, b_{i}=\left|\lambda_{i}\right|$. Then

$$
\left(\sum_{i=1}^{n}\left|\lambda_{i}\right|\right)^{2} \leq\left(\sum_{i=1}^{n} 1\right)\left(\sum_{i=1}^{n}\left|\lambda_{i}\right|^{2}\right)
$$

implying that

$$
[S D D E]^{2} \leq n \cdot 2 P
$$

and hence we get

$$
[S D D E] \leq \sqrt{2 n P}
$$

as an upper bound.

Theorem 3.4. Let $G$ be a graph with $n$ vertices. If $R=\operatorname{det} S D D(G)$, then

$$
\operatorname{SDDE}(G) \geq \sqrt{2 P+n(n-1) R^{\frac{2}{n}}} .
$$

Proof. By definition, we have

$$
\begin{aligned}
(\operatorname{SDDE}(G))^{2} & =\left(\sum_{i=1}^{n}\left|\lambda_{i}\right|\right)^{2} \\
& =\sum_{i=1}^{n}\left|\lambda_{i}\right| \sum_{j=1}^{n}\left|\lambda_{j}\right| \\
& =\left(\sum_{i=1}^{n}\left|\lambda_{i}\right|^{2}\right)+\sum_{i \neq j}\left|\lambda_{i}\right|\left|\lambda_{j}\right| .
\end{aligned}
$$

Using arithmetic-geometric mean inequality, we have

$$
\frac{1}{n(n-1)} \sum_{i \neq j}\left|\lambda_{i}\right|\left|\lambda_{j}\right| \geq\left(\prod_{i \neq j}\left|\lambda_{i}\right|\left|\lambda_{j}\right|\right)^{\frac{1}{n(n-1)}} .
$$

Therefore,

$$
\begin{aligned}
{[\operatorname{SDDE}(G)]^{2} } & \geq \sum_{i=1}^{n}\left|\lambda_{i}\right|^{2}+n(n-1) \cdot\left(\prod_{i \neq j}\left|\lambda_{i}\right|\left|\lambda_{j}\right|\right)^{\frac{1}{n(n-1)}} \\
& \geq \sum_{i=1}^{n}\left|\lambda_{i}\right|^{2}+n(n-1) \cdot\left(\prod_{i \neq j}\left|\lambda_{i}\right|^{2(n-1)}\right)^{\frac{1}{n(n-1)}} \\
& =\sum_{i=1}^{n}\left|\lambda_{i}\right|^{2}+n(n-1) R^{\frac{2}{n}} \\
& =2 P+n(n-1) R^{\frac{2}{n}} .
\end{aligned}
$$

Thus,

$$
\operatorname{SDDE}(G) \geq \sqrt{2 P+n(n-1) R^{\frac{2}{n}}} .
$$

Let $\lambda_{n}$ and $\lambda_{1}$ are the minimum and maximum values of all $\lambda_{i}^{\prime} s$. Then the following results can easily be proven by means of the above results:

Theorem 3.5. For a graph $G$ of order $n$,

$$
\operatorname{SDDE}(G) \geq \sqrt{2 P n-\frac{n^{2}}{4}\left(\lambda_{1}-\lambda_{n}\right)^{2}} .
$$


Theorem 3.6. For a graph $G$ of order $n$ with non-zero eigenvalues, we have

$$
\operatorname{SDDE}(G) \geq \frac{2 \sqrt{\lambda_{1} \lambda_{n}} \sqrt{2 P n}}{\left(\lambda_{1}+\lambda_{n}\right)^{2}} .
$$

Theorem 3.7. Let $G$ be a graph of order n. Let $\lambda_{1} \geq \lambda_{2} \geq \lambda_{3} \geq \ldots \geq \lambda_{n}$ be the eigenvalues in increasing order. Then

$$
\operatorname{SDDE}(G) \geq \frac{\left|\lambda_{1}\right|\left|\lambda_{n}\right| n+2 P}{\left|\lambda_{1}\right|+\left|\lambda_{n}\right|}
$$

\section{Symmetric Division Deg Energy of Some Graph Types}

In this section, we calculate the symmetric division deg energy of some well-known and frequently used graph types including complete, cycle, star, friendship, cocktail party, double star, Dutch windmill, crown and complete bipartite graphs.

Theorem 4.1. The symmetric division deg energy of a complete graph $K_{n}$ is

$$
\operatorname{SDDE}\left(K_{n}\right)=4 n-4 .
$$

Proof. Let $K_{n}$ be the complete graph with vertex set $V=\left\{v_{1}, v_{2}, \ldots, v_{n}\right\}$. For this graph, the symmetric division deg matrix is

$$
S D D\left(K_{n}\right)=\left[\begin{array}{cccccc}
0 & 2 & 2 & \cdots & 2 & 2 \\
2 & 0 & 2 & \cdots & 2 & 2 \\
2 & 2 & 0 & \cdots & 2 & 2 \\
\vdots & \vdots & \vdots & \ddots & \vdots & \vdots \\
2 & 2 & 2 & \cdots & 0 & 2 \\
2 & 2 & 2 & \cdots & 2 & 0
\end{array}\right] .
$$

The characteristic equation then becomes

$$
(\lambda+2)^{n-1}(\lambda-(2 n-2))=0
$$

and the spectrum would be

$$
\operatorname{Spec}_{S D D}\left(K_{n}\right)=\left(\begin{array}{cc}
-2 & 2 n-2 \\
n-1 & 1
\end{array}\right) .
$$

Therefore, $\operatorname{SDDE}\left(K_{n}\right)=4 n-4$.

Theorem 4.2. The symmetric division deg energy of the cycle graph $C_{2 n}$ is

$$
\operatorname{SDDE}\left(C_{2 n}\right)=8+\sum_{m=1, m \neq n}^{2 n-1}\left|4 \cos \frac{\pi m}{n}\right| .
$$

Proof. The symmetric division deg matrix corresponding to the cycle graph $C_{2 n}$ is

$$
S D D\left(C_{2 n}\right)=\left[\begin{array}{cccccccc}
0 & 2 & 0 & 0 & 0 & \cdots & 0 & 2 \\
2 & 0 & 2 & 0 & 0 & \cdots & 0 & 0 \\
0 & 2 & 0 & 2 & 0 & \cdots & 0 & 0 \\
\vdots & \vdots & \vdots & \vdots & \vdots & \ddots & \vdots & \vdots \\
0 & 0 & 0 & 0 & 0 & \cdots & 0 & 2 \\
2 & 0 & 0 & 0 & 0 & \cdots & 2 & 0
\end{array}\right] .
$$

This is a circullant matrix of order $2 n$. Its eigenvalues are

$$
\lambda_{m}= \begin{cases}4, & \text { for } m=0 \\ -4, & \text { for } m=n \\ 4 \cos \frac{\pi m}{n}, & \text { for } 0<m<n, n<m \leq 2 n-1 .\end{cases}
$$

Therefore the symmetric division deg energy is

$$
S D D\left(C_{2 n}\right)=|-4|+|4|+\sum_{m=1, m \neq n}^{2 n-1}\left|4 \cos \frac{\pi m}{n}\right| .
$$

Theorem 4.3. The symmetric division deg energy of the star graph $K_{1, n-1}$ is

$$
\operatorname{SDDE}\left(K_{1, n-1}\right)=2 \frac{n^{2}-2 n+2}{\sqrt{n-1}} .
$$

Proof. Let $K_{1, n-1}$ be the star graph with vertex set $V=\left\{v_{0}, v_{1}, \ldots, v_{n-1}\right\}$ with $v_{0}$ denotes the central vertex. The symmetric division deg matrix is

$$
\operatorname{SDD}\left(K_{1, n-1}\right)=\left[\begin{array}{ccc}
0 & \cdots & \frac{n^{2}-2 n+2}{n-1} \\
\frac{n^{2}-2 n+2}{n-1} & \cdots & 0 \\
\frac{n^{2}-2 n+2}{n-1} & \cdots & 0 \\
\vdots & \ddots & \vdots \\
\frac{n^{2}-2 n+2}{n-1} & \cdots & 0 \\
\frac{n^{2}-2 n+2}{n-1} & \cdots & 0
\end{array}\right] .
$$

The characteristic equation becomes

$$
\lambda^{n-2}\left(\lambda^{2}-\frac{\left(n^{2}-2 n+2\right)^{2}}{n-1}\right)=0
$$

and therefore, the spectrum would have an $\frac{n^{2}-2 n+2}{\sqrt{n-1}}$, $a-\frac{n^{2}-2 n+2}{\sqrt{n-1}}$ and $n-2$ times 0. Therefore, 


$$
\operatorname{SDDE}\left(K_{1, n-1}\right)=2 \frac{n^{2}-2 n+2}{\sqrt{n-1}} .
$$

Definition 4.4. The friendship graph, denoted by $F_{n}^{3}$, is defined as the graph obtained by taking $n$ copies of the cycle graph $C_{3}$ with a vertex in common.

It is clear that $\left|V\left(F_{n}^{3}\right)\right|=2 n+1$.

Theorem 4.5. The symmetric division deg energy of the friendship graph $F_{n}^{3}$ is

$$
\operatorname{SDDE}\left(F_{n}^{3}\right)=4 n-2+\sqrt{\frac{8 n^{4}+16 n^{2}+4 n+8}{n}} .
$$

Proof. Let $F_{n}^{3}$ be the friendship graph with $2 n+1$ vertices and let $v_{0}$ be the common vertex. The symmetric division deg matrix is

$$
\left[\begin{array}{cccccccc}
0 & \frac{n^{2}+1}{n} & \frac{n^{2}+1}{n} & \frac{n^{2}+1}{n} & \frac{n^{2}+1}{n} & \cdots & \frac{n^{2}+1}{n} & \frac{n^{2}+1}{n} \\
\frac{n^{2}+1}{n} & 0 & 2 & 0 & 0 & \cdots & 0 & 0 \\
\frac{n^{2}+1}{n} & 2 & 0 & 0 & 0 & \cdots & 0 & 0 \\
\frac{n^{2}+1}{n} & 0 & 0 & 0 & 0 & \cdots & 0 & 0 \\
\frac{n^{2}+1}{n} & 0 & 0 & 2 & 2 & \cdots & 0 & 0 \\
\vdots & \vdots & \vdots & \vdots & \vdots & \ddots & \vdots & \vdots \\
\frac{n^{2}+1}{n} & 0 & 0 & 0 & 0 & \cdots & 0 & 2 \\
\frac{n^{2}+1}{n} & 0 & 0 & 0 & 0 & \cdots & 2 & 0
\end{array}\right] .
$$

The characteristic equation becomes

$$
(\lambda-2)^{n-1}(\lambda+2)^{n}\left(\lambda^{2}-2 \lambda-2 \frac{\left(n^{2}+1\right)^{2}}{n}\right)=0
$$

implying that the spectrum has $\mathrm{n}$ times $-2, n-1$ times 2, a $1+\sqrt{\frac{2 n^{4}+4 n^{2}+n+2}{n}}$ and a $1-\sqrt{\frac{2 n^{4}+4 n^{2}+n+2}{n}}$. Therefore, we get

$$
\operatorname{SDDE}\left(F_{n}^{3}\right)=4 n-2+\sqrt{\frac{8 n^{4}+16 n^{2}+4 n+8}{n}} .
$$

Theorem 4.6. The symmetric division deg energy of the cocktail party graph $K_{n \times 2}$ is

$$
\operatorname{SDDE}\left(K_{n \times 2}\right)=8 n-8 .
$$

Proof. Let $K_{n \times 2}$ be the cocktail party graph of order $2 n$ having vertex set $\left\{u_{1}, u_{2}, \ldots, u_{n}, v_{1}, v_{2}, \ldots, v_{n}\right\}$. The symmetric division deg matrix is

$$
S D D\left(K_{n \times 2}\right)=\left[\begin{array}{ccccccccc}
0 & 0 & 2 & 2 & \cdots & 2 & 2 & 2 & 2 \\
0 & 0 & 2 & 2 & \cdots & 2 & 2 & 2 & 2 \\
2 & 2 & 0 & 0 & \cdots & 2 & 2 & 2 & 2 \\
2 & 2 & 0 & 0 & \cdots & 2 & 2 & 2 & 2 \\
\vdots & \vdots & \vdots & \vdots & \ddots & \vdots & \vdots & \vdots & \vdots \\
2 & 2 & 2 & 2 & \cdots & 0 & 0 & 2 & 2 \\
2 & 2 & 2 & 2 & \cdots & 0 & 0 & 2 & 2 \\
2 & 2 & 2 & 2 & \cdots & 2 & 2 & 0 & 0 \\
2 & 2 & 2 & 2 & \cdots & 2 & 2 & 0 & 0
\end{array}\right] .
$$

In that case, the characteristic equation is

$$
\lambda^{n}(\lambda+4)^{n-1}(\lambda-(4 n-4))=0
$$

and hence the spectrum becomes

$$
\operatorname{Spec}_{S D D}\left(K_{n \times 2}\right)=\left(\begin{array}{ccc}
4 n-4 & 0 & -4 \\
1 & n & n-1
\end{array}\right) .
$$

Therefore we arrive at the required result:

$$
\operatorname{SDDE}\left(K_{n \times 2}\right)=8 n-8 .
$$

Theorem 4.7. The symmetric division deg energy of the double star graph $S_{n, n}$ is

$$
\operatorname{SDDE}\left(S_{n, n}\right)=2 \sqrt{4+4 \frac{(n-1)\left(n^{2}+1\right)^{2}}{n^{2}}} .
$$

Proof. The symmetric division deg matrix is

$$
\left[\begin{array}{cccccc}
0 & \frac{n^{2}+1}{n} & \cdots & 2 & \cdots & 0 \\
\frac{n^{2}+1}{n} & 0 & \cdots & 0 & \cdots & 0 \\
\frac{n^{2}+1}{n} & 0 & \cdots & 0 & \cdots & 0 \\
\vdots & \vdots & \ddots & \vdots & \vdots & \vdots \\
\frac{n^{2}+1}{n} & 0 & \cdots & 0 & \cdots & 0 \\
2 & 0 & \cdots & 0 & \cdots & \frac{n^{2}+1}{n} \\
0 & 0 & \cdots & \frac{n^{2}+1}{n} & \cdots & 0 \\
\vdots & \vdots & \ddots & \vdots & \vdots & \vdots \\
0 & 0 & \cdots & \frac{n^{2}+1}{n} & \cdots & 0 \\
0 & 0 & \cdots & \frac{n^{2}+1}{n} & \cdots & 0
\end{array}\right]
$$

Hence, the spectrum would have $2 n-4$ times 0 , 
$a-1+\sqrt{1+\frac{(n-1)\left(n^{2}+1\right)^{2}}{n^{2}}}$, and $a-1-\sqrt{1+\frac{(n-1)\left(n^{2}+1\right)^{2}}{n^{2}}}$.

Therefore, we get

$$
\operatorname{SDDE}\left(S_{n, n}\right)=4 \sqrt{1+\frac{(n-1)\left(n^{2}+1\right)^{2}}{n^{2}}} .
$$

Definition 4.8. A graph obtained by joining $n$ copies of the cycle graph $C_{4}$ of length 4 at a common vertex is called a Dutch windmill graph. It will be denoted by $D_{4}^{n}$.

It is clear that the Dutch windmill graph $D_{4}^{n}$ has $3 n+1$ vertices and $4 n$ edges.

Theorem 4.9. The symmetric division deg energy of the Dutch windmill graph $D_{4}^{n}$ is

$$
\operatorname{SDDE}\left(D_{4}^{n}\right)=2 \sqrt{8}(n-1)+\sqrt{50 n+32} .
$$

Proof. Recall that $D_{4}^{n}$ has $3 n+1$ vertices. Then the symmetric division deg matrix is

$$
\left[\begin{array}{ccccccc}
0 & \frac{n^{2}+1}{n} & 0 & \frac{n^{2}+1}{n} & \cdots & 0 & \frac{n^{2}+1}{n} \\
\frac{n^{2}+1}{n} & 0 & 2 & 0 & \cdots & 0 & 0 \\
0 & 2 & 0 & 2 & \cdots & 0 & 0 \\
\frac{n^{2}+1}{n} & 0 & 0 & 0 & \cdots & 0 & 0 \\
\frac{n^{2}+1}{n} & 0 & 0 & 2 & \cdots & 0 & 0 \\
\vdots & \vdots & \vdots & \vdots & \ddots & \vdots & \vdots \\
0 & 0 & 0 & 0 & \cdots & 0 & \frac{1}{\sqrt{2}} \\
\frac{n^{2}+1}{n} & 0 & 0 & 0 & \cdots & 2 & 0
\end{array}\right] .
$$

Hence the characteristic equation will be

$$
(\lambda-\sqrt{8})^{n-1}(\lambda+\sqrt{8})^{n-1} \lambda^{n+1}\left(\lambda^{2}-\frac{25 n+16}{2}\right)=0
$$

and therefore the spectrum would have $n-1$ times $\sqrt{8}$, $n-1$ times $-\sqrt{8}, \quad n+1$ times $0, \sqrt{\frac{25 n+16}{2}}$ and $-\sqrt{\frac{25 n+16}{2}}$. Therefore, it is directly seen that

$$
\operatorname{SDDE}\left(D_{4}^{n}\right)=2 \sqrt{8}(n-1)+\sqrt{50 n+32} .
$$

Theorem 4.10. The symmetric division deg energy of crown graph $S_{n}^{0}$ is

$$
\operatorname{SDDE}\left(S_{n}^{0}\right)=8 n-8 .
$$

Proof. Let $S_{n}^{0}$ be the crown graph of order $2 n$ and let the vertex set of this graph be $\left\{u_{1}, u_{2}, \ldots, u_{n}, v_{1}, v_{2}, \ldots, v_{n}\right\}$.

The symmetric division deg matrix of $S_{n}^{0}$ is

$$
\left[\begin{array}{cccccccccc}
0 & 0 & 0 & \cdots & 0 & 0 & 2 & \cdots & 2 & 2 \\
0 & 0 & 0 & \cdots & 0 & 2 & 0 & \cdots & 2 & 2 \\
0 & 0 & 0 & \cdots & 0 & 2 & 2 & \cdots & 0 & 2 \\
\vdots & \vdots & \vdots & \ddots & \vdots & \vdots & \vdots & \ddots & \vdots & \vdots \\
0 & 0 & 0 & \cdots & 0 & 2 & 2 & \cdots & 2 & 0 \\
0 & 2 & 2 & \cdots & 2 & 0 & 0 & \cdots & 0 & 0 \\
2 & 0 & 2 & \cdots & 2 & 0 & 0 & \cdots & 0 & 0 \\
\vdots & \vdots & \vdots & \ddots & \vdots & \vdots & \vdots & \ddots & \vdots & \vdots \\
2 & 2 & 0 & \cdots & 2 & 0 & 0 & \cdots & 0 & 0 \\
2 & 2 & 2 & \cdots & 0 & 0 & 0 & \cdots & 0 & 0
\end{array}\right] .
$$

Therefore the characteristic equation is

$$
(\lambda-2)^{n-1}(\lambda+2)^{n-1}(\lambda+(2 n-2))(\lambda-(2 n-2))=0
$$

implying that the spectrum has a $2 n-2$, a $2-2 n, n-1$ times 2 and $n-1$ times -2 . Therefore we obtain

$$
\operatorname{SDDE}\left(S_{n}^{0}\right)=8 n-8 .
$$

Theorem 4.11. The symmetric division deg energy of the complete bipartite graph $K_{m, n}$ of order $m \times n$ with vertex set $\left\{u_{1}, \ldots, u_{m}, v_{1}, \ldots, v_{n}\right\}$ is

$$
\operatorname{SDDE}\left(K_{m, n}\right)=2 \frac{m^{2}+n^{2}}{\sqrt{m n}} .
$$

Proof. The symmetric division deg matrix of the complete bipartite graph $K_{m, n}$ is

$\left[\begin{array}{ccccc}0 & 0 & \cdots & \frac{m^{2}+n^{2}}{m n} & \frac{m^{2}+n^{2}}{m n} \\ 0 & 0 & \cdots & \frac{m^{2}+n^{2}}{m n} & \frac{m^{2}+n^{2}}{m n} \\ 0 & 0 & \cdots & \frac{m^{2}+n^{2}}{m n} & \frac{m^{2}+n^{2}}{m n} \\ \vdots & \vdots & \ddots & \vdots & \vdots \\ \frac{m^{2}+n^{2}}{m n} & \frac{m^{2}+n^{2}}{m n} & \cdots & 0 & 0 \\ \frac{m^{2}+n^{2}}{m n} & \frac{m^{2}+n^{2}}{m n} & \cdots & 0 & 0 \\ \frac{m^{2}+n^{2}}{m n} & \frac{m^{2}+n^{2}}{m n} & \cdots & 0 & 0\end{array}\right]$.

Then the characteristic equation is

$$
\lambda^{m+n-2}\left(\lambda-\frac{m^{2}+n^{2}}{\sqrt{m n}}\right)\left(\lambda+\frac{m^{2}+n^{2}}{\sqrt{m n}}\right)=0
$$

and therefore the spectrum has a $\frac{m^{2}+n^{2}}{\sqrt{m n}}, m+n-2$ 
times 0 and $\mathrm{a}-\frac{m^{2}+n^{2}}{\sqrt{m n}}$. Therefore, we get

$$
\operatorname{SDDE}\left(K_{m, n}\right)=2 \frac{m^{2}+n^{2}}{\sqrt{m n}} \text {. }
$$

\section{Symmetric Division Deg Energy of Complements}

Definition 5.1. [5] Let $G$ be a graph and $P_{k}=\left\{V_{1}, V_{2}, \ldots, V_{k}\right\}$ be a partition of its vertex set $V$. Then the k-complement of $G$ is denoted by $\overline{\left(G_{k}\right)}$ and obtained as follows: For all $V_{i}$ and $V_{j}$ in $P_{k}, i \neq j$, remove the edges between $V_{i}$ and $V_{j}$ and add the edges between the vertices of $V_{i}$ and $V_{j}$ which are not in $G$.

Definition 5.2. [5] Let $G$ be a graph and $P_{k}=\left\{V_{1}, V_{2}, \ldots, V_{k}\right\}$ be a partition of its vertex set $V$. Then the $k(i)$-complement of $G$ is denoted by $(G)_{k(i)}$ and obtained as follows: For each set $V_{r}$ in $P_{k}$, remove the edges of $G$ joining the vertices within $V_{r}$ and add the edges of $\bar{G}$ (complement of $G$ ) joining the vertices of $V_{r}$.

There is usually a nice relation between some properties of a graph and its complement. Here we investigate the relation between some special graph classes and their complements in terms of the symmetric division deg energy.

Theorem 5.3. The symmetric division deg energy of the complement $\overline{K_{n}}$ of the complete graph $K_{n}$ is

$$
\operatorname{SDDE}\left(\overline{K_{n}}\right)=0 .
$$

Proof. Let $K_{n}$ be the complete graph with vertex set $V=\left\{v_{1}, v_{2}, \ldots, v_{n}\right\}$. The symmetric division deg connectivity matrix of the complement of the complete graph $K_{n}$ is

$$
S D D\left(\overline{K_{n}}\right)=\left[\begin{array}{cccccc}
0 & 0 & 0 & \cdots & 0 & 0 \\
0 & 0 & 0 & \cdots & 0 & 0 \\
0 & 0 & 0 & \cdots & 0 & 0 \\
\vdots & \vdots & \vdots & \ddots & \vdots & \vdots \\
0 & 0 & 0 & \cdots & 0 & 0 \\
0 & 0 & 0 & \cdots & 0 & 0
\end{array}\right] .
$$

Clearly, the characteristic equation is $\lambda^{n}=0$ implying

$$
\operatorname{SDDE}\left(\overline{K_{n}}\right)=0 \text {. }
$$

Theorem 5.4. The symmetric division deg energy of the complement $\overline{K_{1, n-1}}$ of the star graph $K_{1, n-1}$ is

$$
\operatorname{SDDE}\left(\overline{K_{1, n-1}}\right)=4 n-8 \text {. }
$$

Proof. Let $\overline{K_{1, n-1}}$ be the complement of the star graph with vertex set $V=\left\{v_{0}, v_{1}, \ldots, v_{n-1}\right\}$ where $v_{0}$ is the central vertex. The symmetric division deg matrix is

$$
S D D\left(\overline{K_{1, n-1}}\right)=\left[\begin{array}{cccccc}
0 & 0 & 0 & \cdots & 0 & 0 \\
0 & 0 & 2 & \cdots & 2 & 2 \\
0 & 2 & 0 & \cdots & 2 & 2 \\
\vdots & \vdots & \vdots & \ddots & \vdots & \vdots \\
0 & 2 & 2 & \cdots & 0 & 2 \\
0 & 2 & 2 & \cdots & 2 & 0
\end{array}\right] .
$$

The corresponding characteristic equation is

$$
\lambda(\lambda-(2 n-4))(\lambda+2)^{n-2}=0
$$

and therefore the spectrum is

$$
\operatorname{Spec}_{S D D}\left(\overline{K_{1, n-1}}\right)=\left(\begin{array}{ccc}
-2 & 0 & 2 n-4 \\
n-2 & 1 & 1
\end{array}\right) .
$$

Therefore,

$$
\operatorname{SDDE}\left(\overline{K_{1, n-1}}\right)=4 n-8 .
$$

Theorem 5.5. The symmetric division deg energy of the complement $\overline{K_{n \times 2}}$ of the cocktail party graph $K_{n \times 2}$ of order $2 n$ is

$$
\operatorname{SDDE}\left(\overline{K_{n \times 2}}\right)=4 n .
$$

Proof. Let $K_{n \times 2}$ be the cocktail party graph of order $2 n$ having the vertex set $\left\{n_{1}, n_{2}, \ldots, n_{n}, v_{1}, v_{2}, \ldots, v_{n}\right\}$. The corresponding symmetric division deg matrix is

$$
\left[\begin{array}{ccccccccc}
0 & 0 & 0 & 0 & \cdots & 2 & 0 & 0 & 0 \\
0 & 0 & 0 & 0 & \cdots & 0 & 2 & 0 & 0 \\
0 & 0 & 0 & 0 & \cdots & 0 & 0 & 2 & 0 \\
0 & 0 & 0 & 0 & \cdots & 0 & 0 & 0 & 2 \\
\vdots & \vdots & \vdots & \vdots & \ddots & \vdots & \vdots & \vdots & \vdots \\
2 & 0 & 0 & 0 & \cdots & 0 & 0 & 0 & 0 \\
0 & 2 & 0 & 0 & \cdots & 0 & 0 & 0 & 0 \\
0 & 0 & 2 & 0 & \cdots & 0 & 0 & 0 & 0 \\
0 & 0 & 0 & 2 & \cdots & 0 & 0 & 0 & 0
\end{array}\right]
$$

and the characteristic equation becomes

$$
(\lambda+2)^{n}(\lambda-2)^{n}=0
$$

implying that the spectrum would be

$$
\operatorname{Spec}_{S D D}\left(\overline{K_{n \times 2}}\right)=\left(\begin{array}{cc}
2 & -2 \\
n & n
\end{array}\right) .
$$

Therefore,

$$
\operatorname{SDDE}\left(\overline{K_{n \times 2}}\right)=4 n \text {. }
$$




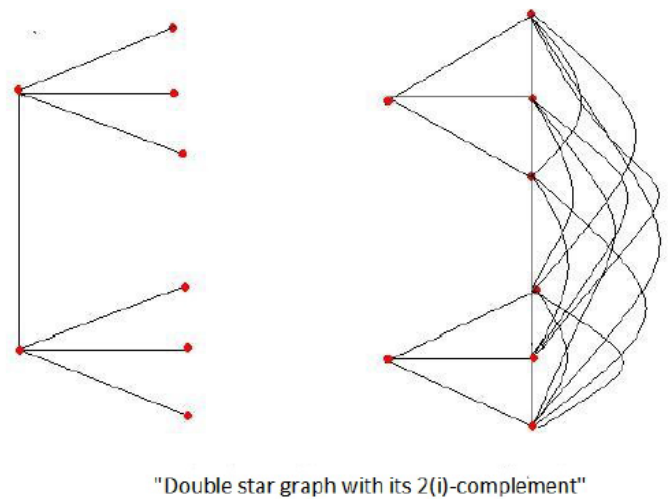

Figure 1. Double star graph with its 2(i)-complement

Theorem 5.6. The symmetric division deg energy of 2(i)-complement of double star graph $S_{n, n}$ is

$$
\operatorname{SDDE}\left(\overline{\left(S_{n, n}\right)_{2(i)}}\right)=2(2 n-4)+A+B
$$

where $A=\sqrt{25 n-21}$ and $B=\sqrt{16 n^{2}-23 n+11}$.

Proof. The symmetric division deg matrix for 2(i)-complement of double star graph is

$$
\left[\begin{array}{cccccccc}
0 & 2.5 & 2.5 & \cdots & 2.5 & 0 & \cdots & 2 \\
2.5 & 0 & 2 & \cdots & 2 & 0 & \cdots & 2 \\
2.5 & 2 & 0 & \cdots & 2 & 0 & \cdots & 2 \\
\vdots & \vdots & \vdots & \ddots & \vdots & \vdots & \vdots & \vdots \\
2.5 & 2 & 2 & 0 & \cdots & 2 & \cdots & 2 \\
0 & 0 & 0 & \cdots & 0 & 0 & \cdots & 2.5 \\
0 & 2 & 2 & \cdots & 2 & 2.5 & \cdots & 2 \\
\vdots & \vdots & \vdots & \ddots & \vdots & \vdots & \vdots & \vdots \\
0 & 2 & 2 & \cdots & 2 & 2.5 & \cdots & 2 \\
0 & 2 & 2 & \cdots & 2 & 2.5 & \cdots & 0
\end{array}\right] .
$$

Therefore the spectrum has $2 n-4$ times -2 , a $\frac{-2+A}{2}$, a $\frac{-2+A}{2}$, a $\frac{4 n-6+B}{2}$ and a $\frac{4 n-6+B}{2}$.

Therefore we obtain the required result.

Theorem 5.7. The symmetric division deg energy of 2-complement of cocktail party graph $K_{n \times 2}$ is

$$
\operatorname{SDDE}\left(\overline{K_{n \times 2(2)}}\right)=8(n-1) .
$$

Proof. Consider the 2-complement of the cocktail party graph $\overline{K_{n \times 2(2)}}$. The symmetric division deg matrix is

$$
\left[\begin{array}{cccccccccc}
0 & 2 & 2 & \cdots & 2 & 2 & 0 & \cdots & 0 & 0 \\
2 & 0 & 2 & \cdots & 2 & 0 & 2 & \cdots & 0 & 0 \\
2 & 2 & 0 & \cdots & 2 & 0 & 0 & \cdots & 2 & 0 \\
\vdots & \vdots & \vdots & \ddots & \vdots & \vdots & \vdots & \ddots & \vdots & \vdots \\
2 & 2 & 2 & \cdots & 0 & 0 & 0 & \cdots & 0 & 2 \\
2 & 0 & 0 & \cdots & 0 & 0 & 2 & \cdots & 2 & 2 \\
0 & 2 & 0 & \cdots & 0 & 2 & 0 & \cdots & 2 & 2 \\
\vdots & \vdots & \vdots & \ddots & \vdots & \vdots & \vdots & \ddots & \vdots & \vdots \\
0 & 0 & 2 & \cdots & 0 & 2 & 2 & \cdots & 0 & 2 \\
0 & 0 & 0 & \cdots & 2 & 2 & 2 & \cdots & 2 & 0
\end{array}\right]
$$

The characteristic polynomial is

$$
\lambda^{n-1}(\lambda+4)^{n-1}(\lambda-(2 n-4))=0
$$

and therefore the symmetric division deg spectrum has $n-1$ times $-4, n-1$ times 0 , a $2 n-4$ and a $2 n$ implying that the symmetric division deg energy is

$$
\operatorname{SDDE}\left(\overline{K_{n \times 2}(2)}\right)=8 n-8 .
$$

\section{Symmetric Division Deg Energy of Graphs with One Edge Deleted}

Edge deletion is very important in combinatorial calculations with graphs. In this section, we obtain the symmetric division deg energy for certain graphs with one edge deleted. This can be used recursively to calculate the symmetric division deg energy of a given graph.

Theorem 6.1. Let e be an edge of the complete graph $K_{n}$. Then $\operatorname{SDDE}\left(K_{n}-e\right)$ is equal to

$$
2 n-6+2 \frac{\sqrt{(n-3)^{2}\left(n^{2}-3 n+2\right)^{2}+(2 n-4)\left(2 n^{2}-6 n+5\right)^{2}}}{n^{2}-3 n+2} .
$$

Proof. The symmetric division deg matrix for $K_{n}-e$ is

$$
\left[\begin{array}{cccc}
0 & 0 & \cdots & \frac{2 n^{2}-6 n+5}{n^{2}-3 n+2} \\
0 & 0 & \cdots & \frac{2 n^{2}-6 n+5}{n^{2}-3 n+2} \\
\frac{2 n^{2}-6 n+5}{n^{2}-3 n+2} & \frac{2 n^{2}-6 n+5}{n^{2}-3 n+2} & \cdots & 2 \\
\vdots & \vdots & \ddots & \vdots \\
\frac{2 n^{2}-6 n+5}{n^{2}-3 n+2} & \frac{2 n^{2}-6 n+5}{n^{2}-3 n+2} & \cdots & 2 \\
\frac{2 n^{2}-6 n+5}{n^{2}-3 n+2} & \frac{2 n^{2}-6 n+5}{n^{2}-3 n+2} & \cdots & 0
\end{array}\right]
$$

Therefore the spectrum would have a

$$
\frac{n-3+\sqrt{(n-3)^{2}\left(n^{2}-3 n+2\right)^{2}+(2 n-4)\left(2 n^{2}-6 n+5\right)^{2}}}{n^{2}-3 n+2},
$$

a $\frac{n-3+\sqrt{(n-3)^{2}\left(n^{2}-3 n+2\right)^{2}+(2 n-4)\left(2 n^{2}-6 n+5\right)^{2}}}{n^{2}-3 n+2}$. $n-3$ times -2 and a 0 , implying the result.

Theorem 6.2. Let $e$ be an edge of the complete bipartite graph $K_{n, n}$. The symmetric division deg energy of $K_{n, n}-e$ is equal to

$$
2 \sqrt{\frac{4 n^{5}+4 n^{4}-20 n^{3}+28 n^{2}-16 n+4}{n^{2}(n-1)}}
$$


Proof. The symmetric division deg matrix for $K_{n, n}-e$ is

$$
\left[\begin{array}{cccc}
0 & 0 & \cdots & \frac{2 n^{2}-2 n+1}{n(n-1)} \\
0 & 0 & \cdots & 2 \\
0 & 0 & \cdots & 2 \\
\vdots & \vdots & \ddots & \vdots \\
0 & \frac{2 n^{2}-2 n+1}{n(n-1)} & \cdots & 0 \\
\frac{2 n^{2}-2 n+1}{n(n-1)} & 2 & \cdots & 0 \\
\frac{2 n^{2}-2 n+1}{n(n-1)} & 2 & \cdots & 0
\end{array}\right] .
$$

Hence, the spectrum would have a $\frac{-2 n+2+A}{2}$, a $\frac{-2 n+2-A}{2}$, a $\frac{2 n-2+A}{2}$, a $\frac{2 n-2-A}{2}$, and $2 n-4$ times 0 implying the result

The following result can easily be proven as above:

Lemma 6.3. Let $K_{1, n-1}$ be the star graph with $n$ vertices and let $e$ be an edge of it. Then $\operatorname{SDDE}\left(K_{1, n-1}-e\right)$ $=\operatorname{SDDE}\left(K_{1, n-2}\right)$ for $n \geq 3$.

\section{Some Open Problems}

Open problem 7.1. With respect to symmetric division deg, determine the class of graphs which are co-spectral and characterize them
Open problem 7.2. With respect to symmetric division deg, determine the class of graphs which are hyperenergetic and characterize them.

Open problem 7.3. With respect to symmetric division deg, determine the class of graphs whose symmetric division deg energy and symmetric division deg energy of their complements are equal.

Open problem 7.4. With respect to symmetric division deg, determine the class of non-co-spectral graphs which are equienergetic.

Open problem 7.5. Determine the class of graphs whose symmetric division deg energy is equal to usual energy.

\section{References}

[1] Alexander, V., Upper and lower bounds of symmetric division deg index, Iranian Journal of Mathematical Chemistry, 5 (2) (2014), 91-98.

[2] Gutman, I., The energy of a graph, Ber. Math. Stat. Sekt. Forschungsz. Graz, 103 (1978), 1-22.

[3] Gutman, I., The energy of a graph: old and new results, Combinatorics and applications, A. Betten, A. Khoner, R. Laue and A. Wassermann, (Eds.), Springer, Berlin, (2001), 196-211.

[4] Randić, M., On characterization of molecular branching, J. Am. Chem. Soc., 97 (1975), 6609-6615.

[5] Sampathkumar, E., Pushpalatha, L., Venkatachalam, C. V. and Bhat, P., Generalized complements of a graph, Indian J. Pure Appl. Math., 29(6) (1998), 625-639.

[6] Todeschini, R., Consonni, V., Handbook of Molecular Descriptors, Wiley-VCH, Weinheim, (2000), 84-90.

[7] Todeschini, R., Consonni, V., Molecular Descriptors for Chemoinformatics, Wiley-VCH, Weinheim, (2009), 161-172.

[8] Zhou, B., Trinajstic, N., On Sum-Connectivity Matrix and Sum-Connectivity Energy of (Molecular) Graphs, Acta Chim. Slov, 57 (2010), 518-523.

[9] Zhou, B., Trinajstic, N., On a novel connectivity index, J. Math. Chem., 46 (2009), 1252-1270. 